\title{
Le Sommet UE-Afrique 2021 \\ Quo vadis, compte tenu du Brexit et de la Covid-19?
}

\author{
Dirk Kohnert ${ }^{1}$
}

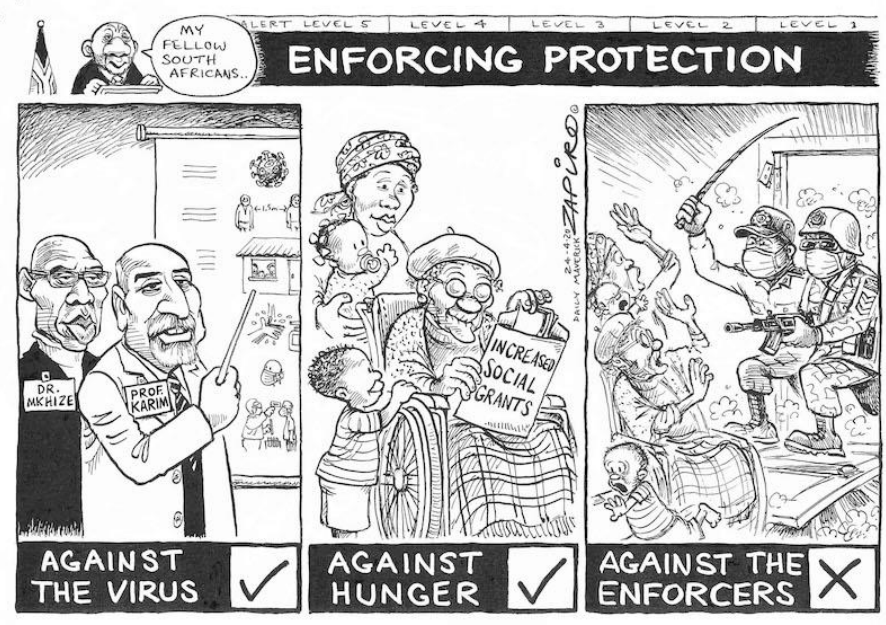

«forcer de la protection»

Source: Zapiro, Daily Maverick, 24 avril 2020

\begin{abstract}
Résumé : Tous les trois ans, le sommet UA-UE réunit les dirigeants africains et européens pour définir l'orientation future de la coopération. Le 6e sommet devait réaffirmer et renouveler le partenariat entre les deux blocs déjà en octobre 2020, mais il a été repoussé au premier trimestre 2021, ou même plus tard, en raison de la crise du COVID-19. En outre, Bruxelles a dû faire face à sa propre situation post-Brexit, compte tenu l'exclusion du Royaume-Uni, et à ses répercussions sur les relations UE-Afrique. Les États africains, pour leur part, souhaitaient renégocier le partenariat UE-Afrique, et l'équilibrer avec les nouvelles visions post-Brexit prometteuses du Premier ministre britannique Johnson sur le renforcement des liens économiques avec l'Anglosphère africaine. La Chine et d'autres acteurs mondiaux sont en concurrence avec l'UE et ses États membre dans la nouvelle ruée vers les ressources africaines. Étant donné que l'Afrique est de plus en plus courtisée par d'autres partenaires, elle pourrait être encline à limiter successivement ses relations avec l'UE et à la considérer comme un simple fournisseur d'aide et de sécurité contre le terrorisme islamique. Cette tendance a été renforcée par le fait que la nouvelle stratégie UE-Afrique n'a toujours pas été approuvée par les États membres de l'UE. Et un remplacement opportun de l'accord de Cotonou, qui expire en novembre 2021, est sujet à caution.
\end{abstract}

Mots clés : Corona, Brexit, Afrique, Royaume-Uni, UE, commerce international, récession économique, pauvreté, violence

JEL-Code: F13, F35, F54, F63, G15, I1, N17, N47, N67, O17, P16, Z13

\section{L'Introduction}

\footnotetext{
${ }^{1}$. Expert associé à l'Institut des affaires africaines, GIGA-Hambourg. Projet, 14 Mars 2021.
} 
Tous les trois ans, le sommet UA-UE réunit les dirigeants africains et européens, en alternance entre les sièges en Afrique et en Europe, pour définir l'orientation future de la coopération. Le 6e sommet avait été de réaffirmer et de renouveler le partenariat entre les deux blocs déjà en octobre 2020 sous la présidence allemande du Conseil de l'UE. Cependant, en raison de la crise du COVID-19, il a été repoussé au premier trimestre de 2021 et plus tard ajourné à juin 2021 (Hermenegildo \& Cunha, 2021). Parce que l'Allemagne a transféré la présidence du Conseil de l'UE au Portugal fin 2020, le Portugal assume la présidence du Conseil de l'UE pour le premier semestre. Entre-temps, il est même devenu incertain si le sommet ne doit pas être reporté une autre fois. En outre, il est permis de douter que la Slovénie, qui présiderait le sommet pour le deuxième semestre, pourrait le gérer avec la même compétence que le gouvernement de Lisbonne, avec son intérêt de longue date dans les affaires africaines. Ce qui suit donne un aperçu à jour de la discussion et de la littérature internationales pertinentes sur le sujet.

Initialement, le sommet était axé sur la renégociation des relations UE-Afrique, y compris un accord de l'Organisation des États d'Afrique, des Caraïbes et du Pacifique (OEACP; c'est-àdire les anciens États membres $\underline{\mathrm{ACP}}$ ) pour remplacer l'accord de Cotonou, signé en 2000 entre l'UE et 79 pays, dont 48 États africains, principalement d'anciennes colonies britanniques et françaises ${ }^{2}$. Cependant, la crise du COVID-19 bouleverse l'agenda ainsi que les priorités des deux partenaires. Par ailleurs, Bruxelles a dû faire face à sa propre situation post-Brexit et à ses répercussions sur les relations UE-Afrique, hors Royaume-Uni. Les États africains, pour leur part, voulaient renégocier le partenariat UE-Afrique et l'équilibrer avec les nouvelles visions post-Brexit prometteuses du Premier ministre britannique Boris Johnson sur le renforcement des liens économiques avec l'Anglosphère africaine.

Même si le cadre politique global des relations UE-Afrique, la stratégie conjointe Afrique-UE (Joint Africa-EU Strategy (JAES)), a été formellement adoptée au sommet de Lisbonne en 2007 par les représentants de 53 États africains et de 27 États européens, et bien que les négociateurs soient parvenus à un accord politique sur un nouvel accord de partenariat postCotonou le 3 décembre 2020, l'UE n'a pas pu profiter de la crise Covid-19 pour souligner la spécificité de ses relations avec le continent africain. Il a même été dépassé par la Chine, qui semblait voler à la rescousse de l'Afrique pendant la pandémie. Le soutien inefficace de l'UE a motivé à nouveau l'Union africaine (UA) à se dépêcher avec la création de la zone de libreéchange continentale africaine (ZLECA) qui devrait lui permettre de négocier des partenariats sur un pied d'égalité avec d'autres dans le monde. Étant donné que l'Afrique est de plus en plus courtisée par d'autres partenaires, elle pourrait être encline à limiter successivement ses relations avec l'UE et à la considérer comme un simple fournisseur d'aide et de sécurité contre le terrorisme islamique.

Cette tendance a certainement été renforcée par le fait que la nouvelle stratégie UE-Afrique n'a toujours pas été approuvée par les États membres de l'UE. En plus, un remplacement opportun de l'accord de Cotonou, qui expire en novembre 2021, est sujet à caution ${ }^{3}$. Bien que

\footnotetext{
${ }^{2}$ L'accord OEACP devra encore être approuvé, signé et ratifié par les parties concernées. Il couvrira un grand nombre de domaines, allant du développement durable et de la croissance aux droits de l'homme, à la paix et à la sécurité, et visera à autonomiser chaque région. Une fois en vigueur, l'accord servira de nouveau cadre juridique et guidera les relations politiques, économiques et de coopération entre l'UE et 79 membres de l' OEACP au cours des vingt prochaines années. L'application de l'ancien accord de Cotonou sera encore prolongée jusqu'au 30 novembre 2021, à moins que le nouvel accord n'entre pas en vigueur ou ne soit appliqué à titre provisoire avant cette date. Commission européenne, Bruxelles, 3 décembre 2020.

3 "L'UE est très préoccupée par elle-même - en partie à cause de la crise du COVID-19", a déclaré Mathias Mogge de VENRO, une organisation faîtière pour les ONG de développement en Allemagne. "Les partenariats
} 
la coopération avec l'Afrique ait gagné en importance dans l'UE face à la politique migratoire et à la concurrence économique et géopolitique croissante avec la Chine, la Russie et d'autres acteurs, les notions d'égalité et d'appropriation partagée, qui avaient été si centrales dans la JAES à sa création, est resté plus une vision qu'une réalité, une grande partie des relations continentales étant toujours fortement tributaire des liens post-coloniaux et de l'argent donné par l'UE (Kell \& Vines, 2020).

L'incident suivant est révélateur du fait que les négociateurs européens ont apparemment dormi à travers les signes des temps. Au vu de la crise corona, les chefs d'État et de gouvernement de l'UE et de l'UA avaient convenu de tenir le sommet au moyen d'une vidéoconférence début décembre 2020. Cependant, la partie africaine a annulé à la dernière minute en raison de divergences sur les questions d'ordre du jour. Les propos provocateurs de deux experts médicaux français au printemps 2020 en sont largement responsables. Ce dernier est apparu dans le contexte de plaintes africaines concernant le traitement actuel et historique des pays et des responsables africains, y compris les problèmes de santé. Les déclarations des experts français sur une éventuelle étude en Afrique sur les effets d'un vaccin antituberculeux dans la lutte contre le coronavirus suggèrent pour la partie africaine que les Africains pourraient également être utilisés comme "cobayes de test" vis-à-vis du vaccin corona (Herszenhorn, 2021). En fait, tester de nouveaux médicaments sur des Africains était une pratique courante dans les sociétés pharmaceutiques européennes depuis l'époque coloniale jusqu'aux années 2000 (Rottenburg, 2009). Cela explique les réserves des médecins et politiciens africains et la demande de relations internationales plus justes entre l'Europe et l'Afrique, y compris l'accès aux vaccins contre la couronne et d'autres médicaments (Flint, 2021).

\section{La nouvelle ruée vers les ressources de l'Afrique}

Un indicateur fort d'une nouvelle ruée vers l'Afrique était la multitude de sommets africains partout dans le monde. Cela concerne non seulement le sommet UE-Afrique et le sommet concurrentiel post-Brexit Royaume-Uni-Afrique 2020, mais aussi le «sommet Chine-Afrique sur le COVID-19 » de juin 2020, le «Sommet de Beijing 2018 du Forum sur la coopération Chine-Afrique », le «sommet Afrique-France $2020 »$, le «sommet Moyen-Orient-Afrique $2020 »$, le «sommet Turquie-Afrique $2020 »$, le «Forum sur la coopération Chine-Afrique (FOCAC) $2021 »$, le « Sommet Russie-Afrique 2019 » et le « Sommet et forum économique Russie-Afrique $2022 »$ (Soulé, 2020). Les dirigeants africains ont utilisé ces plates-formes non seulement comme une opportunité pour développer leur image ou pour attirer des investissements, mais aussi comme un moyen d'exercer davantage d'action au niveau de la politique intérieure. Leurs stratégies ont été inspirées par quatre objectifs principaux:

(1) Attirer les investissements dans un environnement politique international compétitif en devançant leurs partenaires dans l'application de la politique de la scie de mer effectivement prouvée déjà en temps de guerre froide

(2) Diversifier les partenaires économiques et politiques pour réduire la dépendance

(3) Revendiquer leur marge de manœuvre économique, et

avec l'Afrique sont depuis passés au second plan." (Pelz, Daniel (2020): Relations Afrique-Europe: 2020 a été une année perdue. DW, 20.11.2020). 
(4) Échapper à l'isolement politique en obtenant plus de visibilité et en élargissant les réseaux (Soulé, 2020).

L'orientation conventionnelle de la science politique sur la rivalité entre les puissances traditionnelles et émergentes sur le continent a détourné les acteurs africains de leurs propres motivations et stratégies pour choisir leurs partenaires. Par conséquent, une analyse scientifique partisane devrait remettre l'agence africaine au centre et évaluer les Africains en tant qu'agents clé du processus de prise de décision (Soulé, 2020).

\section{Principaux enjeux du sommet UE-Afrique}

Par conséquent, une réinitialisation complète du discours paternaliste sur le partenariat avec l'UE est en retard. Outre la focalisation traditionnelle sur les transformations mondiales, le passage historique de la zone Euro-Atlantique (post-) coloniale à la zone Asie-Pacifique, ainsi qu'une tendance croissante à la multipolarité et à l'intensification de l'interdépendance entre les pays, il y a des besoins immédiats plus urgents en jeu dans les années à venir après Covid19. Ils nécessiteront également une efficacité accrue des politiques et, plus particulièrement, un partenariat sur un pied d'égalité avec des procédures axées sur les résultats, y compris le suivi et l'évaluation (Kaul, 2020).

S'il reste encore de nombreuses questions en suspens, ce qui est certain, c'est que les acteurs africains ne veulent plus être dépassés par la stratégie habituelle de division et de conquête des anciennes puissances coloniales. Au lieu de cela, ils sont de plus en plus favorables à un partenariat «continent à continent» avec l'UE, capitalisant ainsi sur l'agenda 2063 de l'UA, c'est-à-dire le projet de transformation de l'Afrique en puissance mondiale (Forysinski et Emmanuel, 2020).

Ainsi, faire face à la crise Corona façonnera la coopération entre l'UE et l'Afrique pour les années et les décennies à venir. Il informera et structurera également de manière significative la politique UE-Afrique concernant la migration et le développement dans le contexte d'une concurrence géopolitique et économique croissante avec la Chine, les États-Unis, la Russie et d'autres acteurs mondiaux.

La Commission européenne, en collaboration avec le Service européen pour l'action extérieure, a lancé une communication conjointe au Parlement européen et au Conseil sur une nouvelle stratégie UE-Afrique en mars 2020. Elle aborde cinq partenariats distincts, conformément aux priorités, qui serviront comme une base pour de nouvelles discussions lors du 6e sommet UE-Afrique en 2021 (ONUDI, 2020):

(1) Un partenariat pour la transition verte et l'accès à l'énergie

(2) Un partenariat pour la transformation numérique

(3) Un partenariat pour une croissance et des emplois durables

(4) Un partenariat pour la paix et la gouvernance

(5) Un partenariat sur la migration et la mobilité

D'autres points cruciaux à l'ordre du jour du prochain sommet UE-Afrique devraient inclure : 
- L'Agenda 2063 de l'UA - comment changer la donne?

- Comment l'UE peut-elle contribuer au fonctionnement de la Zone de libre-échange continentale africaine (ZLECA)?

- Quel rôle pour la coopération sur la politique climatique internationale?

- Quel rôle d'un Green Deal Europe-Afrique?

- Quel rôle pour la coopération sur la transition énergétique?

- Agenda pour un partenariat UA-UE pour l'investissement

- Vers un partenariat numérique entre l'UE et l'UA, concernant les décideurs politiques nationaux, les entrepreneurs, les innovateurs et les investisseurs, les universités et les groupes de réflexion et les organisations internationales ${ }^{4}$ (DIE, 2020).

\section{Promotion de la démocratie et des droits de l'homme}

La question des droits de l'homme, poussée jusqu'ici par l'UE dans ses relations avec l'Afrique, en contraste frappant avec les relations sino-africaines, devient particulièrement délicate en raison de son possible infliction aux questions de souveraineté et d'agence africaine. Enfin, il appartient aux dirigeants africains eux-mêmes de décider si des «mesures restrictives », telles qu'appliquées dans le passé par l'UE, par exemple concernant la participation très critiquée de certains des dirigeants africains les plus notoires (c'est-à-dire corrompus ou violant les droits humains) tels que Omar el-Bechir (Soudan), Eyadema Gnassingbé (Togo) et Robert Mugabe (Zimbabwe) sont toujours d'actualité. Jusqu'à présent, l'UE a appliqué une approche pragmatique en incluant, plutôt que de sanctionner et d'exclure, ces dirigeants des sommets UE-Afrique (Wyk, 2018). Cependant, chaque élément des «mesures restrictives» influence invariablement les deux partenaires, mais pas nécessairement de la même manière ou dans le même sens, car ils sont liés et s'influencent mutuellement (Kotsopoulos et Mattheis, 2018). Ces enchevêtrements constituent un cadre qui pourrait être utilisé pour recalibrer les perspectives africaines et européennes.

Le rôle des acteurs de la société civile (ASC) dans la relation UE-Afrique est étroitement lié. Cependant, cela nécessiterait de réfléchir au concept d'ASC en tenant dûment compte de ses origines européennes. La manière dont les paramètres descriptifs communs pour comprendre le concept de société civile dans le contexte UE-Afrique devrait être discernés en comprenant, en appréciant, et éventuellement en redéfinissant le concept en appliquant le regard africain en tenant dûment compte de l'impact de la crise Corona et des problèmes de l'UE après le Brexit comme plan pour l'avenir (Madu, 2020).

Reconnaître le poids potentiel des acteurs non-étatiques, y compris les associations professionnelles et les entreprises, dans le façonnement des contours de la mondialisation économique, y compris les relations de pouvoir entre les États et le respect des souverainetés, est devenu obligatoire sous l'impact du COVID-19. Ainsi, la nouvelle ruée vers les ressources de l'Afrique devrait être limitée par le respect du développement durable, de la souveraineté et de la possible renaissance du néo-colonialisme en Afrique, tant au nord qu'au sud du Sahara (Langan et Price, 2020).

\footnotetext{
${ }^{4}$ Les webinaires et documents d'orientation proposés ont été élaborés conjointement par le DIE (Bonn), le Centre africain pour la transformation économique (ACET, Accra), le Centre européen pour les politiques et la gestion du développement (ECDPM, Bruxelles) et le Groupe de réflexion européen (ETTG) . La logique sous-jacente est de rassembler des décideurs africains et européens, des universitaires et des représentants du secteur privé et de la société civile pour identifier les convergences d'intérêt, développer des solutions communes et des opportunités de coopération (DIE, 2020).
} 


\section{Paix et sécurité dans le contexte des relations UE-Afrique}

Le rôle de l'UE dans le contexte de l'architecture de paix et de sécurité de l'Afrique est complexe. L'UE a acquis une perspective plus large de la paix et de la sécurité que les autres acteurs mondiaux qui s'intéressent aux affaires africaines. Les visions européennes allaient audelà de la sécurité militaire et de la lutte contre le terrorisme islamiste. Ils incluaient la sécurité humaine et environnementale ainsi que les questions de migration. Inévitablement, cela affecte également l'agence africaine et la postcolonalité (Steager \& Gwatiwa, 2020). Ainsi, l'UE et ses États membres, notamment la France en tant qu'ancienne puissance coloniale ayant des intérêts dans la soi-disant " France-Afrique », ont préconisé de mêler sécurité, développement et renforcement des institutions au profit des citoyens africains et de la démocratisation. Cette vision européenne s'appuie sur une approche plus holistique, mise en œuvre par exemple comme l'approche globale 2013 de l'UE face aux conflits et crises externes, la stratégie globale 2016 (stratégie globale pour la politique étrangère et de sécurité de l'Union européenne) et le nouveau consensus européen de 2017 sur le développement (Steager \& Gwatiwa, 2020). Il s'inspire largement de la politique onusienne et a été particulièrement promu par les gouvernements occidentaux au début des années 2000, prescrivant des liens croissants entre développement et sécurité au bénéfice mutuel des acteurs européens et africains. L'ONU a également contribué à favoriser une coopération étroite entre les partenaires européens et africains, notamment en matière de sécurité (Steager \& Gwatiwa, 2020). Cependant, pour l'UA, le mantra d'une solution africaine aux problèmes africains a servi le plus souvent d'excuse pour mettre de côté les solutions gênantes de problèmes conflictuels.

\section{Regional integration:}

La promotion de l'intégration régionale constitue un objectif commun des relations entre l'UE et l'Afrique depuis des décennies. L'Agenda 2063 de l'UA lui a donné une impulsion supplémentaire, car il a défini l'intégration régionale comme une première étape nécessaire vers une zone de libre-échange continentale africaine commune (ZLECA; Piccolino, 2020; Kohnert, 2019). La crise Covid-19 a également gravement affecté l'intégration régionale, car tout échec à contenir le virus dans un pays menace en fin de compte la sécurité des autres. Cela appelle à une réponse coordonnée à travers les pays par les Communautés économiques régionales africaines (CER), comme la CEDEAO, l'UEMOA, le COMESA et la SADC pour façonner des réponses régionales collectives et coordonnées. Apparemment, l'UA avait pu inspirer une action collective dans un appel unifié à la solidarité internationale (Medinilla \& Byiers \& Apiko, 2020). Au niveau régional, les réponses à la crise Covid-19 reflètent un large spectre de coopération, allant du partage d'informations à la coordination active des réponses étatiques et des actions collectives. La performance des différentes CER dépendait en dernier lieu, mais non des moindres, de leur histoire régionale et institutionnelle, de caractéristiques structurelles telles que la taille et la cohérence de la CER, ainsi que de la dynamique d'économie politique des pays de sa région. Au moins en Afrique de l'Ouest, les acteurs étatiques et de la société civile ont également profité de l'expérience passée de la gestion de l'épidémie d'Ebola en Afrique de l'Ouest (Medinilla \& Byiers \& Apiko, 2020).

\section{Nécessité d'un nouveau pacte UE-Afrique pour l'emploi}


De plus, un nouveau pacte UE-Afrique pour l'emploi est nécessaire (Rauch, 2021). Les emplois en Afrique ont besoin de conditions commerciales équitables combinées à une politique industrielle axée sur l'emploi et à des initiatives coordonnées des acteurs locaux, ancrées dans le cadre politique, économique et sanitaire de leur région respective et son degré d'infection par le COVID-19. Là encore, une approche régionale transnationale s'impose, car le virus ne tient pas compte des frontières. Cela inclut une politique d'immigration humaine, car la migration africaine augmentera probablement même sous l'impact économique de la crise de Corona sur l'Afrique. Les stratégies de politiques migratoires africaines-européennes communes devraient prendre en compte à la fois: d'une part, le besoin économique permanent d'existence dans de grandes parties de l'Afrique et les efforts qui en résultent pour mettre en place des systèmes de survie translocaux (si nécessaire également intercontinentaux). D'autre part, une politique de migration durable doit être élaborée conjointement, sur la base d'un équilibre des intérêts. Les pierres angulaires importantes des accords de migration UEAfrique devraient être: (1) la lutte contre les causes de la migration, (2) la création de voies d'accès légales, y compris la migration circulaire, même pour les travailleurs peu qualifiés, aux possibilités d'emploi temporaire, et (3) les garanties de retour pour les immigrés clandestins (Rauch, 2020).

\section{Nécessité d'une politique environnementale et agricole commune}

Le changement climatique et son impact sur la population, la politique, l'économie et l'environnement sont également devenus de plus en plus au cœur des relations UE-Afrique. L'agence africaine est marquée par la montée en puissance d'acteurs verts de plus en plus vocaux (Lightfoot, 2020). Cependant, les priorités de ce dernier, par ex. de l'Union africaine (UA), ne sont pas forcément les mêmes que ceux promus par l'UE, notamment via la stratégie conjointe Afrique-UE, adoptée lors du 2e sommet UE-Afrique 2007 à Lisbonne. Les approches européennes actuelles reflètent un déséquilibre de pouvoir plus large dans les relations Afrique-UE, car les règles qui encadrent la relation reflètent celles de l'UE.

Le futur dialogue sur un pied d'égalité pourrait offrir des opportunités à de nouveaux acteurs étatiques et non étatiques en Afrique, ce qui qui à nouveau aura également un impact sur la stratégie de l'UE. La nécessité d'une nouvelle politique agricole et foncière dans les relations UE-Afrique est étroitement liée, bien au-delà de la ruée de longue date pour les ressources africaines. L'extraction des ressources, fondée sur la dépossession et l'exploitation généralisées de la population africaine, était au cœur des relations entre l'Europe et l'Afrique depuis l'avènement du colonialisme européen, dont l'influence perdure jusqu'à nos jours (Lahiff, 2020).

Selon Robert Kappel (2020), deux grands processus de négociation de l'UE avec l'Afrique sont en cours: premièrement, une stratégie globale pour l'Afrique (CSA), et deuxièmement, un remplacement de l'accord de Cotonou qui expire en septembre 2021 (voir ci-dessus). Cependant, le CSA présente des faiblesses conceptuelles. Pas seulement parce que les intérêts européens ne sont que vaguement formulés. Il n'indique pas clairement s'il s'agit d'une action géostratégique à l'égard de la Chine, des États-Unis et des économies émergentes, si l'UE veut simplement promouvoir les investissements européens, ou contribuer au changement climatique, ou aider à éradiquer la pauvreté et le chômage. Le CSA est plutôt un faisceau de visions qui vont de la numérisation, de l'économie verte aux enjeux commerciaux sans les intégrer dans une stratégie. Par conséquent, le CSA doit être révisé de manière significative, en tenant dûment compte du fait que les pays africains et leurs institutions poursuivent leur 
propre programme et que les changements de pouvoir mondiaux sont reconnus (voir cidessus). Les questions ne doivent pas se limiter aux flux financiers, à la restructuration du commerce, à la résolution des crises et des pandémies, à la lutte contre le terrorisme ou à la bonne gouvernance. Il s'agit d'un changement de direction fondamental qui met fin à la dépendance traditionnelle entre l'Europe et l'Afrique. Ainsi, l'engagement européen devrait se concentrer sur de nouveaux domaines, clairement différents des actions géostratégiques de la Chine, de la Russie, des USA et d'autres pays émergents dans la nouvelle ruée vers l'Afrique, c'est-à-dire :

(1) Nouvelle politique agricole,

(2) Accompagnement des processus de transformation,

(3) Lier les investissements directs étrangers à l'entrepreneuriat local

(4) Réorientation des relations commerciales,

(5) Allégement de la dette et restructuration de la zone CFA.

\section{L'impact du Brexit sur les relations UE-Afrique}

Des ambitions élevées de la part des partisans du Brexit et de sombres prédictions de la part des sceptiques ont caractérisé la controverse autour du Brexit. Les cinq questions clé en jeu pour les relations post-Brexit entre la Grande-Bretagne, l'UE et l'Afrique sont les suivantes: l'accès au marché, les investissements directs étrangers (IDE), l'aide, la sécurité et la nature du partenariat. La vision du gouvernement britannique d'une «Grande-Bretagne mondiale » repose largement sur sa coopération renforcée avec les pays du Commonwealth, enfin et surtout dans l'Anglosphère africaine (Gamble, 2021; McDougall, 2019; Wellings, 2019). L'Anglosphère fait partie d'une longue réflexion sur l'union des communautés anglophones du monde entier. L'Anglosphère fait appel à de nombreux Brexiters, car elle leur donne une vision positive de la «Grande-Bretagne mondiale » comme alternative à l'adhésion à l'UE. Pourtant, ce qui est ignoré, c'est le manque de soutien des pays africains anglo-saxons pour des relations beaucoup plus étroites, sauf dans le domaine de la sécurité, et l'incapacité de renforcer les liens économiques à égaler ce que le Royaume-Uni perdra en se séparant de son partenaire économique le plus important, l'UE. Par conséquent, les attentes des Brexiteers et des politiciens africains quant à une relation avec le Commonwealth post-Brexit renforcée et semblable à celle d'un partenariat sont largement infondées. Bien que le Royaume-Uni postBrexit augmentera les choix des pays africains en ce qui concerne les partenaires commerciaux préférés, il reste à se demander si Londres pourrait offrir quelque chose de nouveau que d'autres acteurs mondiaux avec un intérêt croissant pour l'Afrique, comme la Chine, n'ont pas déjà à leur ordre du jour. (Kohnert, 2018).

De plus, le Brexit a des conséquences supplémentaires pour l'Afrique, même une revitalisation du réseau français corrompu et trouble, connu sous le nom de "Françafrique ", qui se développe et se développe à travers le continent, ne peut être exclue (Kohnert, 2019). Alors que l'impact du Brexit sur l'Afrique anglophone était une question majeure dans les discussions britanniques controversées sur les avantages et les inconvénients du Brexit, les répercussions possibles sur l'Afrique francophone avaient rarement été mentionnées. Tout au plus, des effets généraux indirects ont été déclarés, tant pour l'ancien Empire britannique en Afrique que pour les anciennes colonies françaises. D'un autre côté, les Brexiteers britanniques ne se lassent pas d'aspirer au rétablissement de la soi-disant gloire de l'Empire britannique, vraisemblablement basé sur un rapport multi-experts euphorique et trop ambitieux du Africa All-Party Parliamentary Group (APPG, Londres), financé par la Royal Africa Society, sur les relations commerciales et de coopération au développement entre 
l'Afrique et le Royaume-Uni après le Brexit. Bien que l'empire de la soi-disant «Françafrique » ait été dépassé par la domination britannique en Afrique, il est toujours très vivant aujourd'hui. C'est un système politique, militaire, économique et financier intégré de vénalité à travers le continent par lequel les élites politiques et économiques françaises et africaines se grattent le dos au détriment de leurs citoyens. Il est clôturé par une monnaie spéciale, le franc CFA, une concurrence restreinte assurée et des gains extraordinaires pour toutes les parties concernées. Ainsi, la dépendance postcoloniale s'est poursuivie, y compris le pillage des matières premières, avec la complicité de chefs d'État africains corrompus, parfois entretenus à grands frais, mais aussi conscients de leur pouvoir sur l'ancienne métropole. Des exemples sont les escapades du tristement célèbre «empereur» Bokassa de la République centrafricaine dans les années 1970, ou le pillage des ressources pendant des décennies par Omar Bongo au Gabon (réussi après sa mort en 2009 par son fils Ali Bongo) ou du général Eyadéma Ganssingbé. du Togo, poursuivi par l'héritier de son trône, Faure Gnassingbé, toujours au pouvoir. Dans le contexte de l'absence du pouvoir compensateur de la GrandeBretagne au sein de l'UE dans le cas du Brexit, le réseau trouble de la «Françafrique » ou les réseaux alternatifs louches de corruption, de trafic et de blanchiment d'argent en Afrique francophone pourraient se revitaliser et se consolider, notamment dans les pays riches en ressources comme la République démocratique du Congo.

Cependant, le Brexit pourrait également inspirer l'Afrique à relancer un débat sur sa souveraineté, y compris les questions judiciaires et monétaires. Par exemple, concernant la controverse de longue date sur le franc CFA mal adapté et de plus en plus anachronique. En fait, le débat a déjà commencé. Cela a été aidé par des responsables africains de renommée internationale et des critiques du franc CFA, y compris Kako Nubukpo du Togo (ancien chef de la Banque centrale des États de l'Afrique de l'Ouest - $\underline{B C E A O}$ - et ancien ministre togolais), du Sanou Mbaye (Sénégal ; ancien haut fonctionnaire de la Banque africaine de développement), et Carlos Lopez de Guinée-Bissau (ancien secrétaire exécutif de la Commission économique des Nations Unies pour l'Afrique), ainsi que des banquiers africains comme Henri-Claude Oyima (président-directeur général de BGFI Bank). Par ailleurs, depuis quelques années, un mouvement social s'est développé pour réclamer le retrait des Etats africains du CFA. Le 7 janvier 2017, par exemple, une ONG créée et dirigée par le militant Kemi Séba, soutenue par «SOS Pan-Africa », a organisé des manifestations anti-CFA dans plusieurs villes africaines et européennes. Au Sénégal, le groupe «France Dégage » milite pour la «souveraineté monétaire » des pays CFA. Le 20 mai 2020, la France a adopté un projet de loi selon lequel la BCEAO gérant la monnaie des huit nations de l'UEMOA qui utilisent le franc CFA ouest-africain, ne sera plus tenue de déposer la moitié de ses réserves de change auprès du public Trésor de France. En outre, le gouvernement français se retirerait de tous les organes directeurs de la BCEA. C'était la deuxième étape vers la disparition du franc CFA et son remplacement par une monnaie unique appelée « $\underline{\text { Eco }} »$.

\section{L'impact de la pandémie Corona sur les relations UE-Afrique}

Les principaux défis auxquels l'UE est confrontée concernant la pandémie Covid-19 sont le lien étroit entre l'unité et la cohésion internes et la capacité de projeter le pouvoir à l'échelle mondiale. L'UE ne peut entreprendre de manière crédible une rivalité systémique avec des pays comme la Chine et la Russie que si elle peut défendre ses propres valeurs à l'intérieur de ses propres frontières. La pandémie a en outre souligné que l'UE doit travailler sur sa souveraineté stratégique et que son leadership mondial est plus que jamais nécessaire (Koenig \& Stahl, 2020). L'action extérieure de l'UE tend à devenir plus cohésive et efficace au-delà 
des premières phases de réaction à la crise. La comparaison de la gestion de la crise avec des concurrents de l'UE comme les États-Unis, la Chine, la Russie et d'autres acteurs mondiaux montre qu'un pouvoir structurel, réglementaire et normatif renforcé de l'UE sera probablement également nécessaire au lendemain de cette crise. Cela concerne par exemple l'augmentation des investissements dans la recherche et le développement, la diplomatie scientifique active et les réactions rapides, mais aussi durables dans le domaine de la coopération au développement qui incluent des objectifs à long terme de relance sociale, écologique et économique après la crise, et une politique d'information améliorée avec des relations publiques renforcées afin que l'UE puisse maintenir de manière fiable sa position de partenaire le plus important et axé sur le partenariat sur un pied d'égalité pour l'Afrique (Brugner \& Schuch, 2020).

La crise Covid-19 offre une nouvelle opportunité pour l'UE d'établir un partenariat plus équilibré et pragmatique avec l'Afrique, au service de leurs intérêts communs. En particulier, les deux partenaires devront définir de nouveaux mécanismes d'engagement impliquant également le secteur privé et la société civile (Jaidi, 2020). Pourtant, le principal défi sera pour les partenaires de trouver un moyen de résoudre ces problèmes de manière efficace et solidaire, malgré les clivages politiques et les tensions causés par les conflits et la fragilité, encore accentués par la pandémie. Les crises, telles que la lutte contre le terrorisme islamiste au Sahel, la crise de la Corne de l'Afrique et les batailles des chefs de guerre en Libye, ou les retombées économiques et financières de Covid-19, ont créé des divisions qui pourraient compliquer l'issue des processus de partenariat. Il n'est pas improbable que les préoccupations d'acteurs majeurs comme la France, la Russie, la Turquie ou les acteurs régionaux de second rang dominent l'agenda des relations euro-africaines (Jaidi, 2020). Cependant, la crise de Covid-19 offre également l'occasion de penser et d'agir différemment, par ex. concernant la sécurité humaine et la solidarité régionale entre les deux continents. La persistance de la pandémie souligne en outre l'interdépendance des vulnérabilités et la nécessité d'un cadre de partenariat rigoureux, complet et durable. Outre les préoccupations sanitaires et humanitaires, beaucoup ont exprimé des craintes pour la stabilité, la consolidation de l'État et la sécurité de la région. Covid-19 appelle un ensemble de réactions différent de celui habituel. Les aspects à long terme de la crise nécessitent une politique de résilience au-delà des mesures immédiates de soutien économique, social ou financier (Jaidi, 2020). Obligatoire est une approche unie et durable. Tout échec à contenir le virus dans un continent ou un pays menace, en fin de compte, la sécurité des autres (Medinilla \& Byiers \& Apiko, 2020).

\section{Principaux acteurs concurrents: l'effet Chine}

La montée du populisme en Europe, la migration illégale d'Afrique vers l'Europe et les notions croissantes en Afrique d'une Europe condescendante entraînent un changement fondamental dans les relations UE-Afrique dans lesquelles l'ordre mondial est contesté et les principes fondamentaux remis en question (Hodzi, 2020 ). L'Afrique utilise des relations florissantes avec la Chine pour accroître sa puissance et exiger l'égalité. La perception de la concurrence et de la rivalité entre la Chine et l'UE par les dirigeants africains augmente leur pouvoir de négociation dans les négociations avec l'UE.

L'aide chinoise et la coopération politique et économique reposent sur la non-ingérence ainsi que sur le respect de la souveraineté africaine. En outre, Pékin a tendance à mélanger les intérêts commerciaux avec des flux concessionnels, dans un processus d'allocation de l'aide axé sur la demande qui se traduit souvent par des impacts de l'aide locale différents de ceux des donateurs européens (Isaksson \& Kotsadam, 2021). Les programmes chinois de 
coopération économique sont susceptibles d'alimenter la corruption locale, de décourager la participation des syndicats, de diminuer le soutien politique aux titulaires et de rendre les identités ethniques plus visibles dans les zones locales (Isaksson et Kotsadam, 2021).

Ainsi, l'UE est tenue d'adapter sa propre politique africaines pour garder la bonne volonté qu'elle y a acquise (Babarinde, 2020). La stratégie commune Afrique-Europe constitue le cadre du développement, basé sur un discours néolibéral et néo-réaliste pour expliquer la relation durable. Néanmoins, la relation entre l'UE et l'Afrique est encore profondément asymétrique, malgré la rhétorique de l'UE sur la politique de développement à l'égard de l'Afrique qui se situent quelque part entre ses idéaux normatifs et les réalités des cadres alternatifs émergents concurrents (Babarinde, 2020).

Ainsi, l'impact de l'engagement de la Chine en Afrique sur les relations UE-Afrique semble plus pertinent que jamais. L'initiative chinoise «Une ceinture, une route » (OBOR) ou « nouvelle route de la soie », y compris la route maritime de la soie, est censée traverser la côte de l'Afrique de l'Est, où plusieurs ports sont en cours de construction et seront construits. De plus, des succursales ne sont pas seulement construites sur la voie directe de la route maritime de la soie le long de la côte est de l'Afrique via le canal de Suez, mais aussi sur les côtes de l'Afrique centrale jusqu'au golfe du Bénin en Afrique de l'Ouest (Schwerbrock, 2017; Breuer, 2017). Là-bas, des pôles commerciaux chinois sont construits pour la collecte de matières premières et la distribution de produits fabriqués en Chine également comme voies d'accès à l'intérieur des États africains sans littoral, comme le port en eau profonde du Togo, un allié historiquement proche de la Chine. Il est prévu que certains de ces ports soient reliés à l'arrière-pays africain par des routes et des chemins de fer construits par la Chine, construisant ainsi même une deuxième ceinture chinoise sur le continent africain. L'initiative "Une ceinture, une route » ne concerne donc pas seulement l'Eurasie, mais aussi dans une large mesure l'Afrique.

\section{Conclusion}

Dans les relations internationales, les relations UE-Afrique occupent une position unique en raison de l'histoire coloniale partagée des deux partenaires et des racines communes de la culture et de la société qui en résultent, enfin et surtout, une langue commune, y compris la fracture linguistique entre l'Afrique anglophone et francophone. D'une part, cela constitue un argument de vente unique et un différenciateur concurrentiel par rapport aux autres acteurs mondiaux, comme la Chine, les États-Unis et la Russie. Par conséquent, la comparaison du sommet UE-Afrique avec d'autres sommets africains de pays non-membres de l'UE n'a qu'une valeur limitée. D'un autre côté, la complexité des régimes politiques en Afrique a favorisé les comportements politiques fondamentaux, perçus comme une expression spécifique de l'action africaine dans la politique mondiale qui pourrait renforcer leur faible pouvoir dans la politique mondiale, c'est-à-dire le forum-shopping, le changement de régime et la manipulation stratégique des valeurs (Suh I, 2020).

Ainsi, l'Afrique, de plus en plus courtisée par d'autres partenaires, pourrait être encline à limiter successivement ses relations avec l'UE et à considérer les Européens comme un simple pourvoyeur d'aide et de sécurité contre le terrorisme islamique. De plus, le potentiel de renforcement des relations UE-Afrique est limité par la «dépendance à l'égard du chemin suivi » dans laquelle les deux partenaires restent largement piégés (Bossuyt, 2017). Des événements extérieurs, comme le Brexit et la séparation qui en résulte entre le Royaume-Uni et l'UE, se constituant désormais comme un acteur concurrent dans les affaires africaines, 
ainsi que la crise de Corona pourraient offrir de nouvelles perspectives pour sortir de ce piège de la construction «voies de changement ». Cependant, il ne serait pas réaliste de s'attendre à ce que ces changements se concrétisent dans un court laps de temps, car la nouvelle stratégie UE-Afrique n'a toujours pas été approuvée par les États membres de l'UE, et un remplacement opportun de l'accord de Cotonou, qui expire en novembre 2021, est ouvert à la question.

\section{References}

Babarinde, Olufemi (2020): New directions in EU-Africa development initiatives. in: Beringer, Sarah L. \& Sylvia Maier \& Markus Thiel (eds.): EU development policies between norms and geopolitics. London: Springer, International Political Economy Series book series (IPES), pp. 111134

Breuer, Julia (2017): Two belts, one road ? The role of Africa in China's belt \& road initiative. Blickwechsel, July 2017: 1-8

Brugner, Philipp \& Klaus Schuch (2020): The EU's global response to the COVID-19 crisis with a focus on the Eastern Neighbourhood and Africa. Vienna: Österreichische Gesellschaft für Europapolitik, 03 June 2020

Bossuyt, J. (2017): Can EU-Africa relations be deepened? A political economy perspective on power relations, interests and incentives. Brussels: ecdpm, Briefing Note 97

Derek McDougall (2019) English Nationalism, Brexit and the Anglosphere:Wider Still and Wider, The Round Table, 108:6, 725-727 ---- Review of: Wellings, Ben (2019) English Nationalism, Brexit and the Anglosphere:Wider Still and Wider. Manchester: Manchester University Press, 2019, ix $+205 \mathrm{p}$

Flint, Karen (2021): "Africa Isn't a Testing Lab": Considering COVID Vaccine Trials in a History of Biomedical Experimentation and Abuse. Journal of West African History, Vol. 6, Number 2, Fall 2020: $126-140$

Forysinski, W. \& A. Emmanuel (2020): EU-Africa relations - Towards a new comprehensive strategy with Africa. Between a rock an a hard place. Przeglad Strategiczny, 2020.13: 61-78

Gamble, Andrew (2021): The Brexit negotiations and the Anglosphere. The Political Quarterly, 04 January, 2021, online

Hermenegildo, R.S. \& Alice Cunha (2021): Portugal and the 2021 counil preidency: an unambitious programme? Brussels: Trans-European Policy Studies Association, TEPSA-Briefs, January 2021, pp. 1-3

Herszenhorn, David M. (2021): Charles Michel: EU-Africa summit still up in the air. Politico, January 5,2021

Hodzi, Obert (2020): The China effect - African agency, derivative power and renegotiation of EUAfrica relations. In: Haastrup, Toni \& Niall Duggan \& Luís Mah (eds.): The Routledge handbook of EU-Africa relations, London: Routledge, 1st ed., Dec. 31, 2020, pp. 256-265

Isaksson, Ann-Sofie \& Andreas Kotsadam (2021): Chinese Aid to Africa: Distinguishing Features and Local Effects. Munich: cesifo-Forum, 2.2020, Focus: How can Europe better contribute to Africa's economic transformation in a post-Corona era?, July 2020, vol. 21, pp. 27-31

Jaidi, Larabi (2020): The challenges of Euro-African relations in the post-Covid-19 era. Rabat: Policy Center for the New South

Kaul, Inge (2020): Want to Take the Africa-EU Partnership to the Next Level? Press the Reset Button. Munich: cesifo-Forum, 2.2020, Focus: How can Europe better contribute to Africa's economic transformation in a post-Corona era?, July 2020, vol. 21 , pp. 16-21 
Kappel, Robert (2020): Zusammenarbeit Afrika-Europa: Die Chancen zur Neuorientierung nutzen. (blog)

Keijzer, Niels, \& Alfonso Medinilla (2017): Can the EU prioritise both the African Union and the Africa, Caribbean and Pacific group? European Think Tanks Group, Brussels: ECDPM, November 2017

Kell, Fergus \& Alex Vines (2020): The evolution of the Joint Africa- EU Strategy (2007-2020). In: Haastrup, Toni \& Niall Duggan \& Luís Mah (eds.): The Routledge handbook of EU-Africa relations, London: Routledge, 1st ed., Dec. 31, 2020, pp. 105- 120

Köhler, Horst (2020): Crisis and credibility - Towards new honesty in EU-Africa relations. CESifo Forum, München, ifo Institut, Vol. 21, Iss. 02, pp. 11-15

Koenig, Nicole \& Anna Stahl (2020): How the coronavirus pandemic affects the EU's geopolitical agenda. Hertie School, Jacques Delors Centre, IPolicy Paper, 24 April 2020

Kohnert, Dirk (2019a): Brexit and the Revival of Françafrique. ROAPE-blog. London: Review of African Political Economy (ROAPE), 19 June 2019

Kohnert, Dirk (2019): The ethics of African regional and continental integration. MPRA WP, No.95562

Kohnert, Dirk (2018): More equitable British post-Brexit Africa relations: doomed to fail?, Africa Spectrum, vol. 53., No. 2, pp. 119-130

Kotsopoulos, John \& Frank Mattheis (2018): A contextualisation of EU-Africa relations: Trends and drivers from a reciprocal perspective, South African Journal of International Affairs, 25:4, 445-460

Lahiff, Edward (2020): Agriculture and land in EU-Africa relations. In: Haastrup, Toni \& Niall Duggan \& Luís Mah (eds.): The Routledge handbook of EU-Africa relations, London: Routledge, 1st ed., Dec. 31, 2020, pp. 336-348

Lightfoot, Simon (2020): Environment and climate change in the context of EU-Africa relations. In: Haastrup, Toni \& Niall Duggan \& Luís Mah (eds.): The Routledge handbook of EU-Africa relations, London: Routledge, 1st ed., Dec. 31, 2020, pp. 314 -323

Madu, Uzoamaka (2020): The role of civil society in EU- Africa relations . In: Haastrup, Toni \& Niall Duggan \& Luís Mah (eds.): The Routledge handbook of EU-Africa relations, London: Routledge, 1st ed., Dec. 31, 2020, pp. 324-335

Maurice, Paul (2021): Presidence Allemande du Conseil de 1'UE : Quel bilan geopolitique ?, GéopoWeb.fr, 4 février 2021. Paul Maurice is research fellow at the Comité d'études des relations franco-allemandes.

Medinilla, Alfonso \& Bruce Byiers \& Philomena Apiko (2020): African regional responses to COVID-19. Brussels: ecdpm, DP No. 272, May 2020

Pelz, Daniel (2020): Africa-Europe relations: 2020 was a lost year. Bonn: DW, 20.11.2020

Piccolino, Giulia (2020): Regional integration. In: Haastrup, Toni \& Niall Duggan \& Luís Mah (eds.): The Routledge handbook of EU-Africa relations, London: Routledge, 1st ed., Dec. 31, 2020, pp. $188-201$

Langan, Mark \& Sophia Price (2020): Toward a post-Westphalian turn in Africa-EU studies? Nonstate actors and sustainable development. In: Haastrup, Toni \& Niall Duggan \& Luís Mah (eds.): The Routledge handbook of EU-Africa relations, London: Routledge, 1st ed., Dec. 31, 2020, pp. 282-292

Rauch, Theo (2021): Für einen afrikanisch-europäischen Beschäftigungspakt. (blog) Weltneuvermessung,

Rauch, Theo (2020): Europäische Einwanderungspolitik: Jenseits von Zweckoptimismus und Panikmache (blog) Weltneuvermessung 
Rottenburg, Richard (2009): Social and public experiments and new figurations of science and politics in postcolonial Africa, Postcolonial Studies, 12:4, 423-44

Schwerbrock, Julia (2017): Two Belts, One Road? The Role of Africa in China's Belt \& Road initiative. ResearchGate, July 2017

Soulé, Folashade (2020): 'Africa+1' summit diplomacy and the 'new scramble' narrative: Recentering African agency. African Affairs, Vol. 119, Issue 411, October 2020, pp. 633-646

Staeger, Ueli \& Tshepo T. Gwatiwa (2020): Peace and security in the context of EU-Africa relations. In: Haastrup, Toni \& Niall Duggan \& Luís Mah (eds.): The Routledge handbook of EU-Africa relations, London: Routledge, 1st ed., Dec. 31, 2020, pp.

Suh I, Norbert (2020): International Regime Complexity in EU-Africa Relations. The Journal of Territorial and Maritime Studies, Vol. 7, No. 2 (Summer / Fall 2020), pp. 27-48

UNIDO (2020): COVID-19 Impact on EU -Africa Relations. Brussels: UNIDO-Focus, June 2020

Wellings, B (2019): English Nationalism, Brexit and the Anglosphere: Wider Still and Wider (New Perspectives on the Right), Manchester University Press, 23. Mai 2019

Wyk, Jo-Ansie van (2018): Sanctions and summits: Sanctioned African leaders and EU-Africa summits. South African Journal of International Affairs, 2018, Vol. 25, No. 4, 497-515 
Abstract: The EU-Africa summit 2021 : Quo vadis, in the light of Brexit and Corona ? Every three years, the AU-EU summit reunites African and EU leaders to outline the future direction of cooperation. The 6th summit had been to reaffirm and renew the partnership between the two blocks already in October 2020, but it was pushed back to the first quarter of 2021 or even later due to COVID-19 crisis. Besides, Brussels had to deal with its own postBrexit situation and its repercussions on EU-Africa relations, excluding the UK. African states, for their part, wanted to renegotiate the EU-Africa partnership and to balance it with new promising Post-Brexit visions of the British premier Johnson about increased economic ties with the African Angloshere. China and other global players compete with the EU and its member states in the new scramble for African resources. Given that Africa is increasingly courted by other partners it could be inclined to successively limit its relations with the EU and see it as a mere provider of aid and security against Islamic terrorism. This trend was reinforced by the fact that the new EU-Africa strategy still hasn't been approved by EU member states. And a timely replacement of the Cotonou Agreement, which expires in November 2021, is open to question.

Zusammenfassung: Der EU-Afrika Gipfel: Quo vadis, angesichts des Brexit und der Corona Krise?

Alle drei Jahre bringt der EU-Afrika Gipfel die Staats- und Regierungschefs Afrikas und der EU zusammen, um die künftige Richtung der Zusammenarbeit zu skizzieren. Das 6. Gipfeltreffen sollte bereits im Oktober 2020 die Partnerschaft zwischen den beiden Blöcken bekräftigen und erneuern, es wurde jedoch aufgrund der COVID-19-Krise auf das erste Quartal 2021 oder sogar auf später verschoben. Darüber hinaus musste sich Brüssel mit seiner eigenen Situation nach dem Brexit und dessen Auswirkungen auf die Beziehungen zwischen der EU und Afrika ohne Großbritannien auseinandersetzen. Die afrikanischen Staaten iherseits wollten die Partnerschaft zwischen der EU und Afrika neu verhandeln und sie mit neuen vielversprechenden Visionen des britischen Premierministers Johnson nach dem Brexit über verstärkte wirtschaftliche Beziehungen zur afrikanischen Anglosphäre in Einklang bringen. China und andere Global Player konkurrieren mit der EU und ihren Mitgliedstaaten im erneuten Kampf um afrikanische Ressourcen. Angesichts der Tatsache, dass Afrika zunehmend von anderen Partnern umworben wird, könnte es dazu neigen, seine Beziehungen zur EU sukzessive einzuschränken und die EU als bloßen Anbieter von Hilfe und Sicherheit gegen den islamischen Terrorismus $\mathrm{zu}$ betrachten. Dieser Trend wurde noch dadurch verstärkt, dass die neue EU-Afrika-Strategie von den EU-Mitgliedstaaten noch nicht gebilligt wurde. Ein rechtzeitiger Ersatz des Cotonou-Abkommens, das im November 2021 ausläuft, ist fraglich. 